\title{
Use of Mesh based Variance Reduction Technique for Shielding Calculations of the Stellarator Power Reactor HELIAS
}

\author{
André Häußler ${ }^{\mathrm{a}}$, Ulrich Fischer ${ }^{\mathrm{a}}$, Felix Warmer ${ }^{\mathrm{b}}$ \\ ${ }^{a}$ Karlsruhe Institute of Technology (KIT), Institute for Neutron Physics and Reactor Technology (INR), Hermann-von- \\ Helmholtz-Platz 1, 76344 Eggenstein-Leopoldshafen, Germany \\ ${ }^{b}$ Max Planck Institute for Plasma Physics (IPP), Wendelsteinstr. 1, 17491 Greifswald, Germany
}

\begin{abstract}
The shielding capability is an important aspect of a fusion power plant. The neutron flux decreases in areas far away from the plasma chamber while at the same time the statistical error in Monte Carlo particle transport simulations increases significantly in such regions. This requires variance reduction methods to guide the particles in regions of interest and improve the statistical accuracy. The mesh based weight window technique, applied with ADVANTG, is investigated in this paper and successfully applied for the first neutronic investigation of the shielding performance for the HELIAS stellarator. The obtained results are in an area with high neutron wall load and reduced material thickness. They will be evaluated against the design requirements specified for the EU DEMO tokamak fusion reactor. The results show that the current HELIAS design cannot fulfill the shielding limits specified for DEMO in the investigated area, which is mainly due to the limited space available in the stellarator and should be overcome by improved design solutions for blanket and shield.
\end{abstract}

Keywords: HELIAS; CAD; Neutronics; Variance Reduction; DAGMC

\section{Introduction}

The Helical-Axis Advanced Stellarator (HELIAS) is a conceptual design of a fusion power reactor proposed by the Max Planck Institute for Plasma Physics (IPP) in Greifswald, Germany. HELIAS-5B is a specific 5-fieldperiod concept using the Deuterium-Tritium fusion reaction with a fusion power of $3000 \mathrm{MW}$ [1]. A thorough neutronic design analysis has to be performed on this stellarator in order to provide the input required for the reactor design.

A stellarator confines the fusion plasma with external magnetic fields only produced by non-planar shaped modular field coils, which are important to generate the rotational transform of the magnetic field in the plasma chamber. This type of fusion reactor represents a challenging task for the design and maintenance of technological components such as the breeder blanket and the radiation shield as outlined in Figure 1.

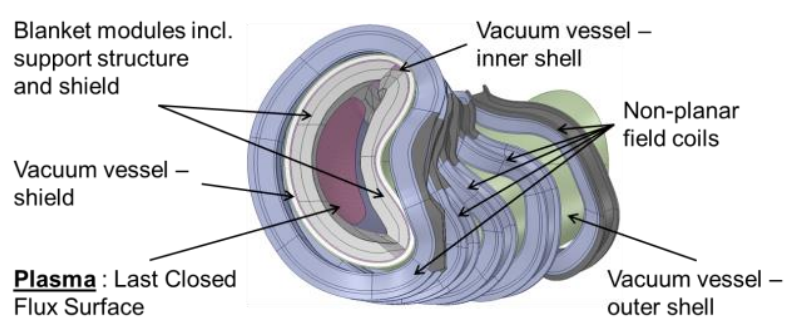

Figure 1: HELIAS-5B CAD model including material layers and last closed flux surface [2].

The standard approach to develop geometry models for neutronics design analyses is using Computer-Aided Design (CAD) tools. The developed CAD models are typically not directly applicable for Monte Carlo (MC) particle transport codes and need preprocessing with regard to the geometrical simplification and adaptation to the requirements of neutronic simulations including the decomposition of complex CAD models [3]. Three different CAD to MCNP geometry conversion approaches were previously investigated with a simplified stellarator geometry showing that all three approaches generate consistent neutronic results [2]. The most suitable way to process the complex CAD model of HELIAS is employing the Direct Accelerated Geometry Monte Carlo (DAGMC) method [4]. The DAGMC code is an extension to the Monte Carlo N Particle (MCNP) transport code [5] in order to allow using complex surface descriptions, like spline surfaces, directly in the simulation.

The purpose of this paper is to investigate the use of mesh based importance sampling technique for $\mathrm{MC}$ shielding calculations in an area of interest of the HELIAS stellarator. At the end, reliable results for the neutron flux, nuclear energy deposition and radiation damage are obtained in a critical area of the stellarator geometry with a high neutron wall load of $\sim 1.4 \mathrm{MW} / \mathrm{m}^{2}$ and reduced material thickness of $\sim 100 \mathrm{~cm}$. The results are evaluated against the EU DEMO tokamak fusion reactor design requirements.

\section{HELIAS geometry}

The current HELIAS model is constructed with a layered structure, filled with homogenized materials. As tritium self-sufficiency is an important criterion for a fusion power reactor, HELIAS is filled with a homogenized material mixture of the Helium Cooled Pebble Bed (HCPB) breeder blanket [6]. This breeder concept needs less space compared to other concepts in order to generate a sufficient amount of tritium [7]. The currently used radial layered construction and their thicknesses are described in Table 1. The JEFF 3.2 
nuclear data library [8] is used in the neutronic calculations.

Table 1. Radial build of HELIAS.

\begin{tabular}{|c|c|c|}
\hline Name & $\begin{array}{l}\text { Thickness } \\
{[\mathrm{cm}]}\end{array}$ & $\begin{array}{l}\text { Material } \\
\text { Composition }\end{array}$ \\
\hline Tungsten Armor & 0.2 & $100 \%$ Tungsten \\
\hline First Wall & 2.5 & $\begin{array}{l}70 \% \text { Eurofer [9], } \\
30 \% \text { Helium }\end{array}$ \\
\hline Breeder Zone & 50 & $\begin{array}{l}\text { HCPB with } 60 \% \\
\text { Li- } 6 \text { enrichment }\end{array}$ \\
\hline $\begin{array}{l}\text { Back Support } \\
\text { Structure (BSS) }\end{array}$ & $\sim 10-40$ & $\begin{array}{l}75 \% \text { Eurofer, } \\
25 \% \text { Helium }\end{array}$ \\
\hline $\begin{array}{l}\text { Vacuum Vessel } \\
\text { (VV) inside }\end{array}$ & 6 & $\begin{array}{l}100 \% \text { Steel } \\
(\mathrm{SS}-316)\end{array}$ \\
\hline VV shield & 20 & $\begin{array}{l}60 \% \text { Steel (SS- } \\
316), 40 \% \text { Water }\end{array}$ \\
\hline VV outside & 6 & $\begin{array}{l}100 \% \text { Steel } \\
(\mathrm{SS}-316)\end{array}$ \\
\hline
\end{tabular}

In Table 1 can be seen that most material layers have a fixed thickness, except the BSS. The complex geometry of the stellarator, related to the shape of the non-planar shaped field coils, has a different total thickness at every point in poloidal and toroidal direction. Therefor the BSS has a different thickness everywhere and fills up the space between breeder zone and vacuum vessel.

\section{Weight window generation}

The superconducting magnetic field coils are key components for a fusion reactor, and they are very sensitive for neutron radiation. The neutron wall load (NWL) distribution of HELIAS shows that there are areas with very high loads [7]. Such an area can be seen in the yellow circle in Figure 2, which has a total thickness of $103 \mathrm{~cm}$.

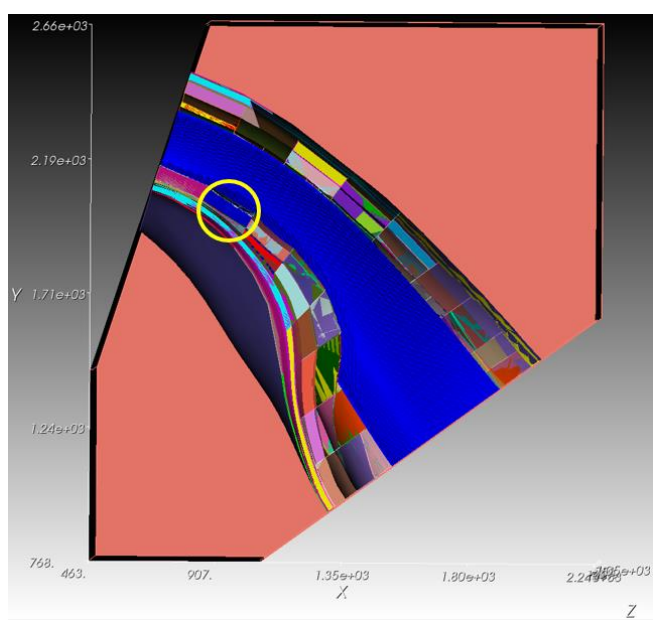

Figure 2: HELIAS cross-section at the mid-plane including bounding box (orange), and highlighted is the area of interest at the inboard (yellow circle)

With analog calculation, without applying the variance reduction (VR) method, the neutron flux in areas far away from the plasma chamber decreases with an increasing of the relative statistical error at the same time. In MC calculations, results with a relative statistical error $\leq 10 \%$ are considered reliable, except for point detectors [5]. The application of the cell based importance sampling technique for a simplified model showed that this technique has potential for application to the HELIAS reactor [10]. A drawback of this method is that it requires the splitting of the geometry into reasonable numbers of layers, which is very difficult in case of the real stellarator geometry. Therefore, the application of the VR method based on a weight window (WW) mesh is studied in this paper.

Two different approaches for generating a suitable WW are investigated, the MCNP internal weight window generator [5] and the application of the AutomateD VAriaNce reducTion Generator (ADVANTG) [11]. The MCNP WW generator estimates the adjoint flux through a forward simulation, while ADVANTG calculates the adjoint flux in an analytical way. Both approaches need a superimposed mesh that covers the whole geometry. A WW mesh is obtained with a weight lower-bound value on each mesh cell, while the upper bound is provided in the MCNP input card as a factor, usually 5.0, to the lower bound. When the weight of a particle is above/within/below the weight window cell, the particle entering this mesh cell will be split/kept/killed by Russian Roulette with the particle weight adjusted accordingly.

The MCNP internal WW generator can be applied directly to the complex DAGMC geometry, as well as the user source subroutine [12] that describes the stellarator neutron source. It needs several iterations on the whole generation process and the WW must be tested at each step if it is sufficient for the intended use. This method is tedious and the achievement of the WW generator highly depends on the specified WW mesh setup. Because one DAGMC simulation needs a huge amount of CPU time and memory, the iteration process is not efficient. Therefore, this WW generation method is not considered in this work.

On the other hand, ADVANTG needs only one run for the WW generation. However, a major drawback of ADVANTG is that it only supports traditional computational solid geometry (CSG) and the fixedsource representation with MCNP's SDEF card. Therefore, a simplified CSG model was created for HELIAS, containing only one material layer with a volume weighted mixture based on the real HELIAS representation. The last closed flux surface (LCFS) was used as boundary for a volumetric source, sampling 14 $\mathrm{MeV}$ neutrons homogeneously. The results obtained with the simplified model would be almost the same as with a more detailed model due to the nature of the analytical calculation.

Two different WW mesh settings were investigated, a uniform mesh with a size of $20 \times 20 \times 20 \mathrm{~cm}^{3}$ and a nonuniform mesh with a fine representation of $5 \times 5 \times 5 \mathrm{~cm}^{3}$ in the region of interest and a coarser representation in the other regions. Both meshes have a total number of $\sim 10^{6}$ elements. Higher numbers caused troubles during the WW generation hence this limit was used. ADVANTG 
needs a target mesh tally for the WW generation, which is shown in the Figures 3 and 4 in blue, including the two different WW mesh settings. The calculated WW will guide more particles to this target region and reduce the computational time on simulating other unconcerned regions, thus accelerating the MC simulation. An additional mesh tally is utilized in this region from first wall to the outside. It has a size of $10 \times 10 \times 1 \mathrm{~cm}^{3}$ while the $1 \mathrm{~cm}$ steps are in radial direction.
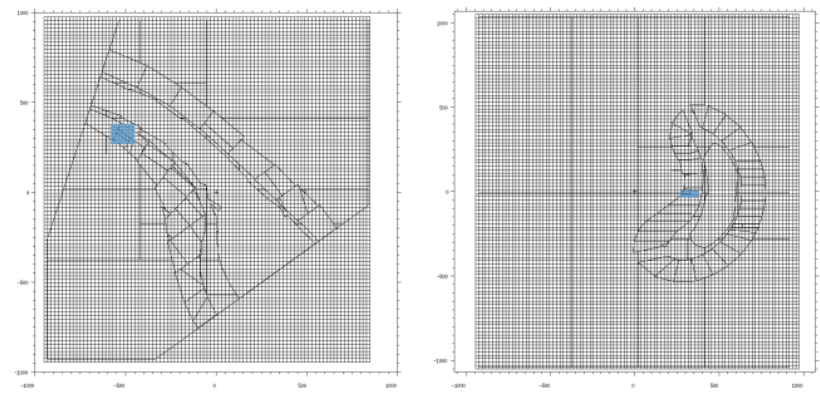

Figure 3: Uniform WW mesh in two different cross sections with the ADVANTG area of interest (blue)
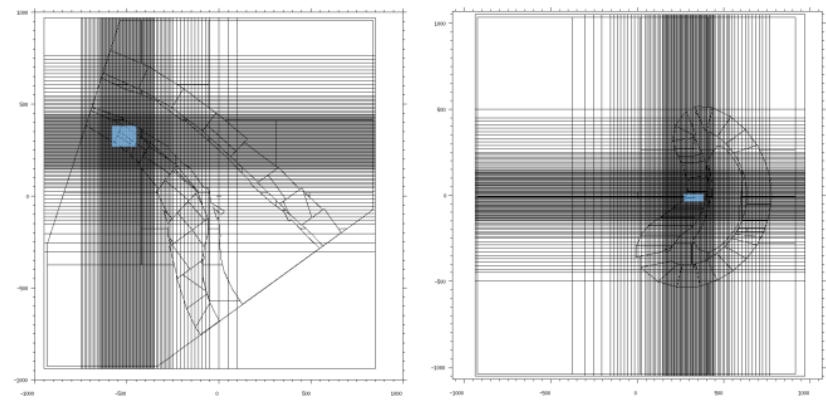

Figure 4: Non-uniform WW mesh in two different cross sections with the ADVANTG area of interest (blue)

Different WW optimization are generated in ADVANTG, neutron optimization for both WW and coupled neutron-photon optimization only for the nonuniform mesh. As MCNP test case the energy deposition tally was used, separated for neutrons and photons, with a fixed number of source particles. The computational time of the non-VR case was $\sim 0.5$ day, for all VR cases $\sim 1$ day. The computational resources, like number of cores and memory, stayed the same for each calculation. In Figure 5 the relative statistical errors obtained in the different runs is shown.

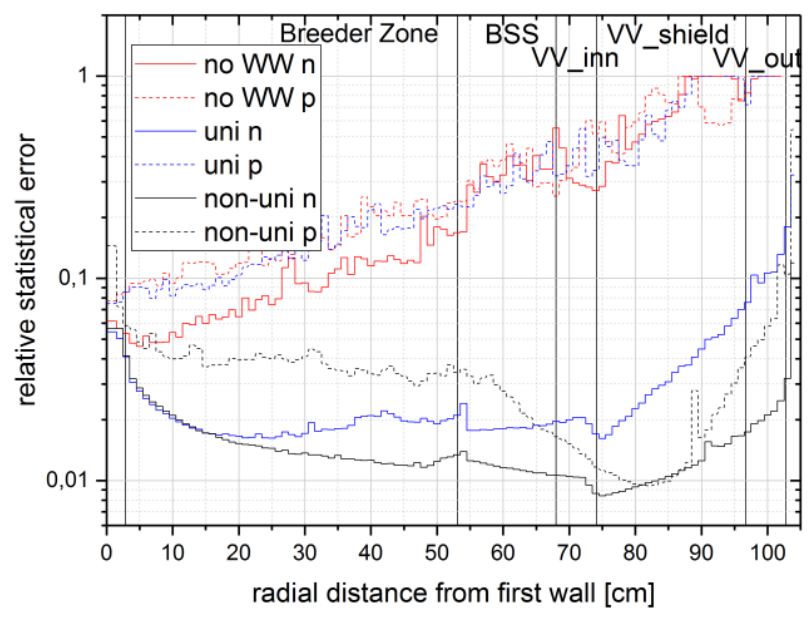

Figure 5: Relative statistical error as function of the radial distance from first wall for three different WW scenarios, neutron error in continuous and photon error in dotted lines. Here "uni" represents the uniform and "non-uni" the nonuniform WW mesh.

Figure 5 shows significant improvements obtained with the applied WW mesh. Comparing the uniform and non-uniform WW meshes shows that the latter one performs better in both neutron and photon flux calculations. An important outcome of this investigation is that both neutron and photon WW should be generated in order to obtain good and reliable statistics.

\section{Computation and Results}

The neutronic analyses include calculations of the neutron flux, the nuclear power density / nuclear heating and the radiation damage. The DEMO design requirements $[13,14]$ are useful for evaluating the results. The mesh grid of the target mesh tally, which was used for the WW qualification, is applied with different tally multiplication cards to compute all nuclear responses of interest.

The neutron flux is recorded in the low and high energy range with the boundaries set to $0.1 \mathrm{MeV}$, as well as a sum of both as total flux. The radial profiles are shown in Figure 6 from the first wall down to the front of the magnet. There is a target for the neutron flux density included according to the DEMO tokamak operation scenario and should be not exceed in order to limit the neutron fluence, based on the DEMO irradiation scenario, to the superconducting magnetic field coils $[13,14]$.

The radial profile of the nuclear heating, including neutron and photon contributions, is shown in Figure 7. According to the DEMO tokamak specifications [13,14], the peak power density in the winding pack of the magnets should be less than $50 \mathrm{~W} / \mathrm{m}^{3}$ to limit the cooling requirements to a reasonable magnitude.

The displacement damage to the steel of the vacuum vessel, which is a lifetime component, should be less than $2.75 \mathrm{dpa}$ (displacements per atom). This is to ensure that the fracture toughness is reduced by not more than $30 \%$ and thus the structural functionality of the vessel can be guaranteed over the entire lifetime [13]. Figure 8 
shows the radial profile of the dpa to iron, calculated based on the NRT damage model for one full power year.

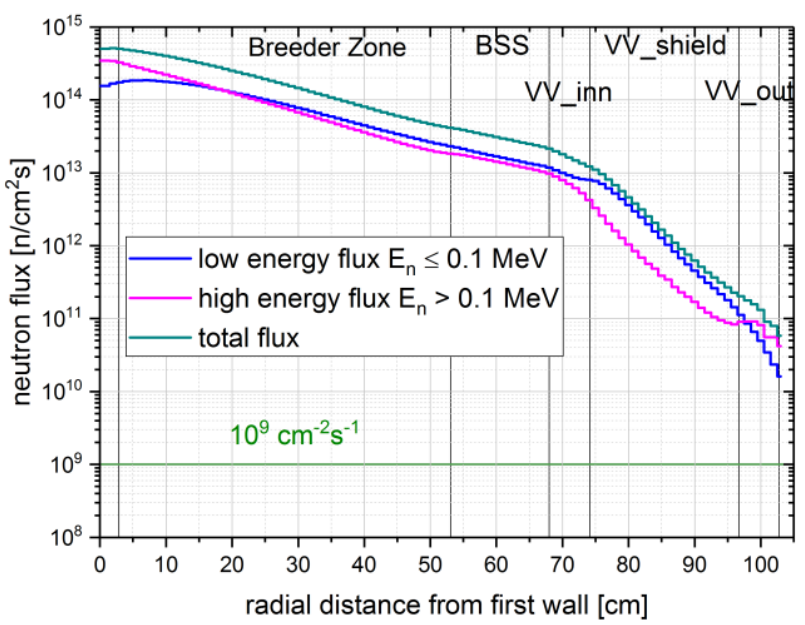

Figure 6: Radial profile of the neutron flux including the DEMO target neutron flux of $10^{9} \mathrm{~cm}^{-2} \mathrm{~s}^{-1}$ to the superconducting magnetic field coils.

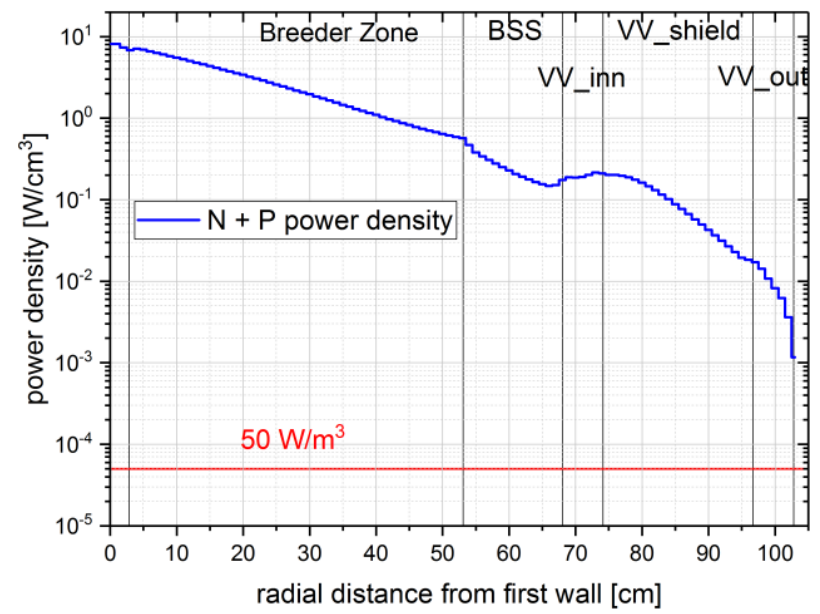

Figure 7: Radial profile of the nuclear power density including the DEMO design limit of the peak power density in the winding pack of the magnets should of $50 \mathrm{~W} / \mathrm{m}^{3}$.

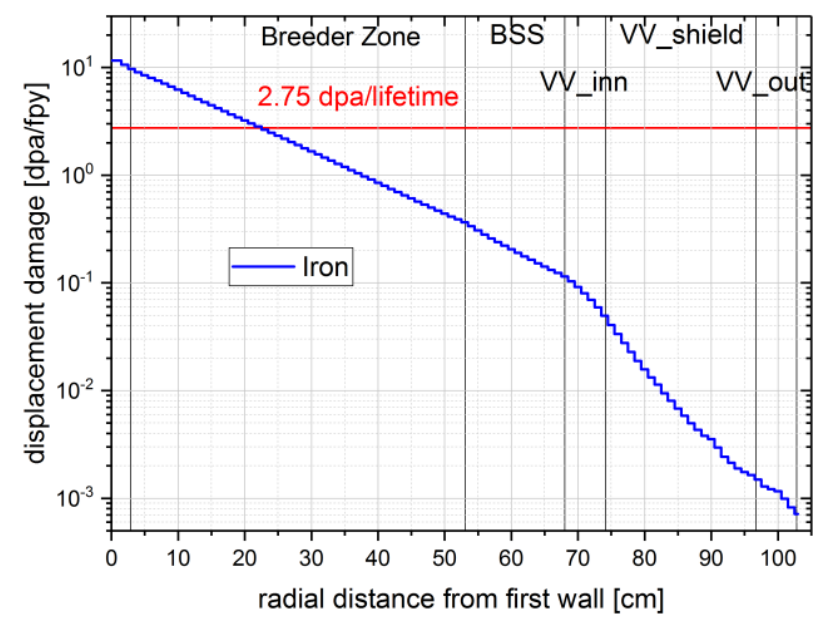

Figure 8: Radial profile of the displacement damage to iron calculated for one full power year including DEMO lifetime design limit of 2.75 dpa for the vacuum vessel.
As seen in Figures 6 and 7, the results obtained for the selected regions in HELIAS exceed significantly the radiation load limits specified for the DEMO tokamak. This is mainly due to the limited space available at this location for the shielding. Possible measures to ensure sufficient shielding performance with the present HELIAS configuration, compliant with the DEMO tokamak specifications, are to increase the thickness of the shielding zone at the expense of the breeder zone, and/or utilize more efficient shielding material, such as tungsten combined with water.

The displacement damage level obtained for the front of the vacuum vessel is $\sim 0.11 \mathrm{dpa} / \mathrm{fpy}$. This would guarantee operating HELIAS for 25 full power years (fpy) until the DEMO design limit of $2.75 \mathrm{dpa}$ is reached.

\section{Conclusion and Outlook}

A detailed shielding analysis has been performed for HELIAS based on MC calculations with the MCNP code. A suitable variance reduction methods based on weight window meshes generated with ADVANTG were utilized in the calculations. A detailed study on improving the WW mesh quality has been conducted in order to improve the neutron and photon flux statistics and provide reliable results across the entire blanket/shield system down to the superconducting magnetic field coils.

The results show that the current HELIAS design cannot fulfill the shielding requirements specified for the DEMO tokamak reactor, at the selected regions of the stellarator. This is mainly due to the limited space available and should be overcome by improved design solutions for blanket and shield.

\section{Acknowledgments}

This work has been carried out within the framework of the EUROfusion Consortium and has received funding from the Euratom research and training programme 2014-2018 under grant agreement No 633053. The views and opinions expressed herein do not necessarily reflect those of the European Commission.

This work was performed on the computational resource ForHLR II funded by the Ministry of Science, Research and the Arts Baden-Württemberg and DFG ("Deutsche Forschungsgemeinschaft").

\section{References}

[1] F. Schauer, et al., HELIAS 5-B magnet system structure and maintenance concept, Fusion Engineering and Design 88 (2013), pages 1619-1622, ISSN 0920-3796

[2] A. Häußler, et al., Verification of different Monte Carlo approaches for the neutronic analysis of a stellarator, Fusion Engineering and Design (2017), volume 124, pages 1207-1210, ISSN 0920-3796

[3] L. Lu, et al., Improved algorithms and advanced features of the CAD to MC conversion tool McCad, Fusion Engineering and Design 89 (2014), pages 1885-1888, ISSN 0920-3796

[4] P. Wilson, et al., Acceleration techniques for the direct use 
of CAD-based geometry in fusion neutronics analysis, Fusion Engineering and Design 85 (2010), pages 17591765, ISSN 0920-3796

[5] X-5 Monte Carlo Team, MCNP - Version 5, Vol. I: Overview and Theory, LA-UR-03-1987 (2003)

[6] F. Hernández, et al., A new HCPB breeding blanket for the EU DEMO: Evolution, rationale and preliminary performances, Fusion Engineering and Design volume 124 (2017), pages 882-886, ISSN 0920-3796

[7] A. Häußler, et al., Neutronics analyses for a stellarator power reactor based on the HELIAS concept, Fusion Engineering and Design 136 (2018), pages 345 - 349, ISSN 0920-3796

[8] JEFF, JEFF-3.2 evaluated data library - Neutron data, https://www.oecdnea.org/dbforms/data/eva/evatapes/jeff 32/n-1-H001.jeff32, Release March 2014

[9] EFDA, Final Report on the EFDA Article 7 contract EFDA/06-1903 on Procurement of reduced activation ferritic-martensitic steel type 9CrWTaV (EUROFER) for the TBM fabrication technology trials and mock-ups, Tech. Rep., July 2009, Saarschmiede GmbH

[10] A. Häußler and U. Fischer, Use of the Cell Based Importance Sampling Technique in Neutronics Shielding Calculations of a Simplified Stellarator Model, Compact at the 49th Annual Meeting on Nuclear Technology (AMNT 2018), 2018, Berlin, Germany

[11] S. Mosher, et al., ADVANTG An Automated Variance Reduction Parameter Generator, ORNL/TM-2013/416, Rev. 1 (2015)

[12] A. Häußler, et al., Neutronics source modeling for stellarator power reactors of the HELIAS-type, Compact at the $47^{\text {th }}$ Annual Meeting on Nuclear Technology (AMNT) 2016, Hamburg, Germany

[13] U. Fischer, et al., Neutronics requirements for a DEMO fusion power plant, Fusion Engineering and Design 9899 (2015), pages 2134-2137

[14] C. Bachmann. et al., Overview over DEMO design integration challenges and their impact on component design concepts, Fusion Engineering and Design 136 (2018), pages 87 - 95, ISSN 0920-3796 